\title{
Perceived numerosity of spatiotemporal events
}

\author{
JÜRI ALLIK \\ University of Tartu, Tartu, Estonia \\ and University of Jyuaskyla, Jyvaskyla, Finland \\ and \\ TIIA TUULMETS \\ University of Tartu, Tartu, Estonia
}

\begin{abstract}
Numerosity discrimination was examined when items were varied in space-time position rather than in space only. Observers were instructed to indicate which of two adjacent streams of visual events contained more items. The precision of numerosity discrimination of dynamic events was not remarkably different from that of static patterns. Two basic numerosity biases previously found for static dot patterns-inhibitory overestimation and satellite underestimation-were demonstrated for items distributed randomly over a spatiotemporal interval. It was also demonstrated that two streams, equated in the number and luminous energy of items, are not judged equal in their visible number if items in one of these two streams have longer duration than items in the second stream. These findings can be accounted for by the occupancy model of perceived numerosity (Allik \& Tuulmets, 1991a) if it is supposed that the impact that each element has on its neighborhood is spread along both spatial and temporal coordinates. Perceived numerosity decreases with both spatial and temporal proximity between the visual items. Space and time have interchangeable effects on perceived numerosity: the amount of numerosity bias caused by the spatial proximity of items can also be produced by the properly chosen temporal proximity of items.
\end{abstract}

The human observer is able to estimate the approximate number of items even without direct one-by-one counting. This ability, however, is neither very precise nor unbiased. One well-established bias is the dependence of the perceived number on the spatial configuration of dots. In principle, the expected distance between two nearest neighbors in a random distribution of dots can be made larger (inhibitory processes) or smaller (satellite processes) than that in a completely random bombarding of a certain plane area with dots (see Diggle, 1983; Ripley, 1981). These two opposite changes lead to two opposite numerosity biases: the inhibitory overestimation and the satellite underestimation (Allik \& Tuulmets, 1991a; cf. Ginsburg, 1976; Ginsburg \& Goldstein, 1987). In the first type, the inhibitory pattern, dots are arranged as if they were animals defending their personal territories. These patterns typically appear more numerous than a completely random (Poisson) distribution of the same number of dots. In the second type, the satellite pattern, each dot appears to attract some other dots, and, on the aver-

This project was started during the first author's visit at the Department of Psychology, University of Nijmegen, which was supported by a grant from that institution. The authors thank Eric Helsper and Piet Vos for fruitful discussions of this research topic. The comments of Norman Ginsburg and two anonymous reviewers on an earlier version of the manuscript were very helpful. We are extremely grateful to Lester Krueger for many valuable suggestions and excellent editorial help. Correspondence concerning this article may be sent to J. Allik, Department of Psychology, University of Tartu, 78 Tiigi Street, Tartu 2400, Estonia. age, the nearest neighbor is closer than it would be in a completely uniform distribution. These patterns look less numerous than random patterns generated without restrictions on the spatial distances between their elements.

The illusory under-and overestimations of numerosity have often been considered to be mistakes made by the visual system. There is, however, a more plausible interpretation of these biases. Morgan, Hole, and Glennerster (1990) have argued that the visual system is highly constrained in the nature of the judgments that it is able to make. In particular, this means that one can formulate relatively easily a verbal instruction that the visual system is not able to carry out exactly. If that is so, numerosity "illusions" may result not from misestimations, but from a failure to carry out the required estimation. Instead of the numerosity as such, another stimulus attribute, which only indirectly corresponds to the actual number of items in the stimulus, is estimated.

A plausible candidate for the stimulus attribute on which the observer's decisions about the numerosity is based is the stimulus area apparently occupied by all elements. Each element, typically a dot, is supposed to have an influence on its neighborhood in the constant radius $R$. The area of the stimulus plane occupied collectively by all dots is the stimulus attribute that is actually judged when the subject is instructed to estimate the numerosity of items; the pattern with the larger occupancy value is chosen as more numerous (Allik \& Tuulmets, 1991a). The occupancy model provides a common explanation for numerosity biases, inhibitory overestimation, and satellite underestima- 
tion. If two dots are less than a distance $2 R$ apart, their individual territories overlap, and their total contribution is reduced proportionally to the size of overlap. Since the overlap in the satellite pattern is expected to be larger than that in a completely random distribution, the satellite patterns appear to be less numerous than random ones. In an inhibitory pattern, in turn, individual territories overlap less than they do in a random pattern and numerosity ought to be overestimated in relation to its estimation with a completely random distribution of dots.

Research in the area of numerosity perception has been based almost exclusively on static patterns (see Allik \& Tuulmets, 1991b; Luccio, 1983). The only temporal parameter, the duration of exposition, that was studied does not change the static character of patterns used (Horne \& Turnbull, 1977; Hunter \& Sigler, 1940; Lechelt \& Nelson, 1971). Indeed, there is another tradition, which consists of the study of temporal numerosity, but it is typical in that field to use a series of flashes delivered at one and the same spatial position. The only model proposed to explain the discrimination of temporal numerosity contains no parameters describing spatial relations between stimulus elements (Viviani, 1979). Consequently, both spatial and temporal domains of the numerosity perception have been studied, but only in isolation from each other. Only in the tactile domain has the problem of the numerosity-based discrimination of spatiotemporal patterns attracted some interest (Lechelt, 1974a, 1974b).

In the present study, we investigated the ability to discriminate numerosity in two assemblies whose items varied in their spatiotemporal position rather than in space only. We will demonstrate that the generalization from static patterns to a time-varying sequence of events occurred without a remarkable change in sensitivity, which may indicate that the judgments about numerosity of spatiotemporal items are not very different from those of static patterns. This conclusion is also supported by the discovery of the inhibitory overestimation and the satellite underestimation of spatiotemporal events.

\section{GENERAL METHOD}

The experiments consisted of trials in which two constantly changing streams of visual events were presented in the left and right hemifields of the display. Figure 1 illustrates the basic stimulus configuration used in this study. The stimulus consisted of two horizontally separated imaginary rectangular areas on the dark background of a VGA color monitor, within each of which light dots could appear. At a distance of $70 \mathrm{~cm}$, the horizontal separation between these two rectangular areas was approximately $5.4^{\circ}$. The size of each rectangular area was approximately $3.2^{\circ}$ in width and height; the size of each dot was about $0.023^{\circ}$. In all cases (except Experiment 3 ), the luminance of the dot when turned on was equivalent to about $41.2 \mathrm{~cd} / \mathrm{m}^{2}$ when a larger uniform test field was turned on and measured by a luminance meter. Each stimulus field contained a regular set of $30 \times 30$ available spatial locations. The horizontal separation between two locations that could be occupied by dots was about $0.093^{\circ}$.

The whole stimulus presentation time was divided into 40 successive frames $(f)$ during each of which the dot patterns on the display did not change. The duration of each frame was $40 \mathrm{msec}$, during which the whole display was refreshed twice. The whole sequence lasted $1,600 \mathrm{msec}$ and contained $36,000(30 \times 30 \times 40)$ spatiotemporal positions in each stream, which served as a skeleton for visual items. Each visual item started with the appearance of a luminous dot in some spatial position and ended $f$ frames later with the disappearance of the dot. Within a given stream, all items had equal duration and equiprobable location within the spatiotemporal frame of positions. Two items could not occupy the same spatial and temporal positions. Because items usually lasted several frames, there were no sequences of static patterns: dots appeared and disappeared at different times and places, giving the impression of a continuous flow of items. The series of overlapped squares (Figure 1) demonstrate the sequence of frames between which dots could appear or disappear from the screen. There were no series of static patterns. Some of the existing dots disappeared and some new dots appeared in new locations at various time moments

One of the two streams (i.e., the standard stimulus) was randomly chosen before each trial as a reference containing a fixed number of reference items. The number of test items in the second of the two streams (i.e., the comparison stimulus) was systematically varied around the reference number. The observer's task was to press one of two buttons indicating which of the two streams, the left or the right, contained more dots. The responses were always coded in terms of the test choice probability and plotted as the percent of cases when the test stream was preferred to the reference stream. The number of items in the test stream varied in a manner that produced a full psychometric function-that is, with an increase in the number of test items, the choice frequency increased smoothly from about $0 \%$ to about $100 \%$. Data points in the empirical psychometric function were approximated by a cumulative Gaussian function using the method of direct approximation. ${ }^{1}$ There was a consistent asymmetry between the discrimination of numerosity increments and decrements, but it was negligible.

Two measures characterizing the sensitivity and bias were derived from these functions. The slope of the function (measured as the distance between the $50 \%$ and $75 \%$ points) indicates the observer's ability to discriminate between the number of items in the test and reference streams. Sensitivity measured from the slope of the psychometric function corresponds to the classical concept of the just noticeable difference (JND). The observer's bias was measured by the location on the psychometric function at which the test and reference stream choice probabilities were equal to $50 \%$. This value is the point of subjective equality (PSE). If the bias is expressed in relative terms as the difference between the PSE and the number of reference items, it will be called the constant error (CE). The goodness of fit was estimated by the mean approximation error (MAE): the mean deviation of a data point from the best fitting psychometric function. If the MAE did not exceed the mean standard error (MSE), it was regarded as acceptable.

Every experiment was divided into five successive sessions, within each of which each stimulus condition was presented 20 times, and the sequence of trials was completely randomized. In total, each
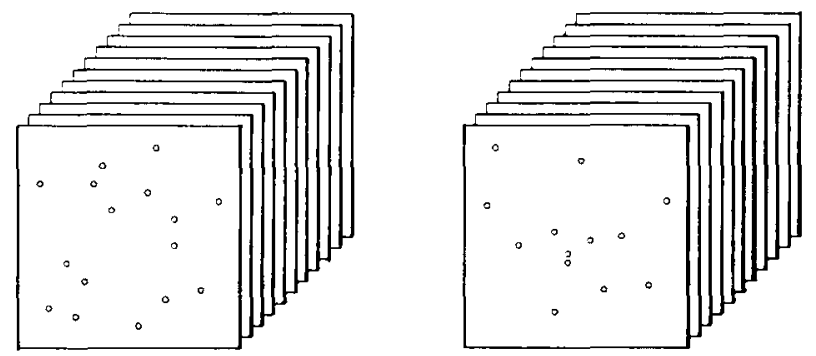

Figure 1. Schematic view of stimuli used in this experiment. The square areas indicate regions of display where visual items, symbolized by small circles, could appear. 
data point shown in the figures and corresponding to one definite stimulus condition is an average of 100 single trials.

Three highly experienced observers, including the two authors, with normal or appropriately corrected myopic vision, participated in the experiments. Viewing was binocular without head fixation, in a semidarkened room. The observer was instructed to fixate the central fixation mark between two stimulus sequences. No feedback about correctness of the choice was provided.

\section{EXPERIMENT 1}

\section{Method}

The main purpose of this experiment was to test how sensitivity depends on the number of reference items. There were four different values of reference items: $n=30,60,90$, and 150 . The number of test items varied on 9 levels symmetrically around these 4 reference values, giving 36 different test stimulus values. All items in both streams lasted $f=8$ frames or $d=320 \mathrm{msec}$-that is, an average of one fifth of the total number of items shown in each stream was visible at one time.

\section{Results}

The choice frequencies of 2 observers are plotted in Figures $2 \mathrm{~A}$ and $2 \mathrm{~B}$, as a function of the number of items in
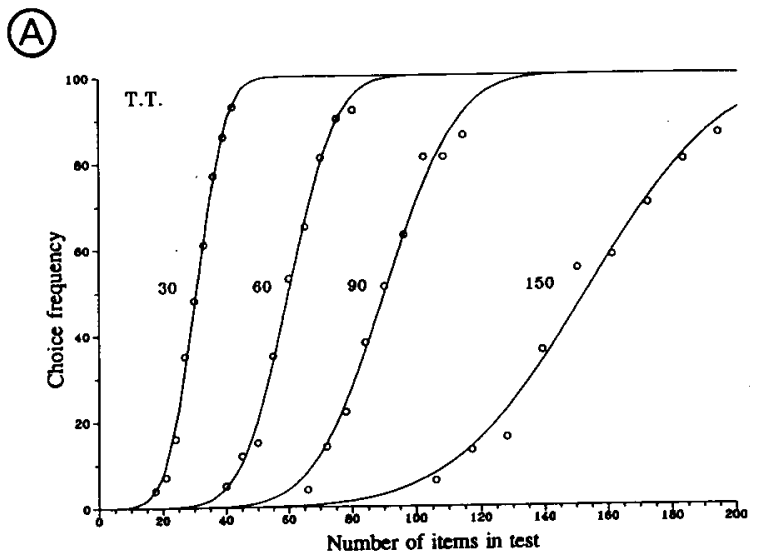

(B)

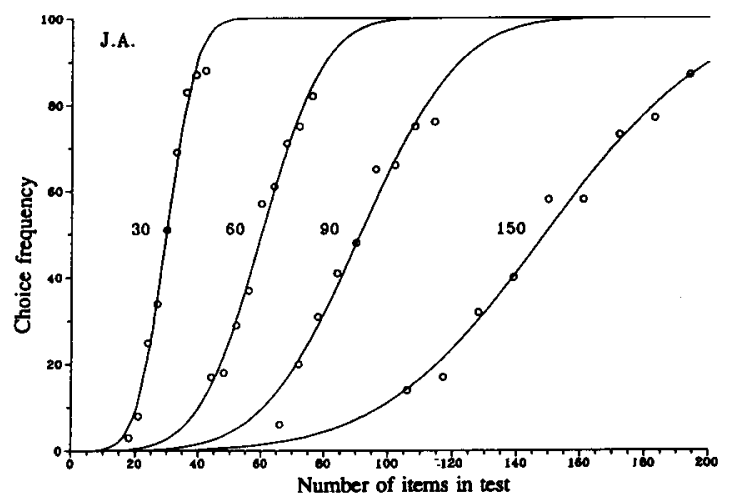

Figure 2. The choice frequency of test stimulus for 2 observers, T.T. (A) and J.A. (B), as a function of the number of items $(n)$ in the test stream and the number of items in the reference stream, $n=30,60,90$, and 150 . Each data point is an average of 100 trials. The best-fitting psychometric functions are shown by continuous curves.
Table 1

Parameter Values of the Best-Fitting Functions Shown in Figure 2

\begin{tabular}{rrrrrr}
$r$ & \multicolumn{1}{c}{ PSE } & \multicolumn{1}{c}{ JND } & JND $/ n$ & MSE (\%) & MAE (\%) \\
& \multicolumn{5}{c}{ T.T. (Figure 2A) } \\
30 & 30.6 & 5.0 & .167 & 4.01 & 1.96 \\
60 & 60.0 & 8.0 & .133 & 4.62 & 2.42 \\
90 & 90.2 & 11.6 & .129 & 4.05 & 3.43 \\
150 & 153.2 & 22.5 & .150 & 4.10 & 4.00 \\
& \multicolumn{5}{c}{ J.A. (Figure 2B) } \\
30 & 29.8 & 5.0 & .166 & 3.86 & 3.38 \\
60 & 60.0 & 10.5 & .175 & 4.41 & 3.29 \\
90 & 91.3 & 16.1 & .179 & 4.40 & $4.57^{*}$ \\
150 & 148.9 & 27.2 & .181 & 4.34 & 3.28
\end{tabular}

Note $-n$, number of reference items; PSE, point of subjective equality; JND, just noticeable difference; JND/ $n$, Weber fraction; MSE, mean standard error (percent); MAE, mean approximation error (percent). *MAE > MSE.

the test stream. Continuous curves are the best fits to the experimental data. The fit was generally quite satisfactory, since in all but one case the MAE was smaller than the MSE: the mean deviation of a data point from the bestfitting function was smaller than its standard error (Table 1). In only one case did the MAE exceed the MSE (Observer J.A., $n=90$ ), and, even in this worst case, the Pearson product moment correlation between the observed and predicted data was acceptable $(r=.981$, $p<.0001$ ).

The slope of the psychometric function decreased with the number of reference items. If about 5 additional items produced a noticeable difference from 30 reference items, then 22-27 extra items were required to notice the difference from 150 reference ones (see Table 1). The observer's sensitivity to changes in the numerosity was nearly invariant when expressed in terms of the Weber fraction, JND $/ n$. Over the whole range tested, the Weber fraction was .144 and .175 for Observers T.T. and J.A., respectively. These values are typical of a static numerosity discrimination performance (see Allik \& Tuulmets, 1991a). Moreover, both observers have participated as subjects in previous experiments. For example, Observer T.T.'s discrimination of the numerosity of two 400-msec static patterns yielded Weber fractions of .152 $(n=20)$ and $.133(n=40)$, which is rather close to the Weber fraction of .144 obtained in the present study (Allik \& Tuulmets, 1991a). Both observers were also subjects in another study, in which their Weber fractions were .160 (Allik, Tuulmets \& Vos, 1991; the case of the equal-sized patterns). Consequently, there are no remarkable differences in sensitivity in the numerosity discrimination of static and dynamic patterns. A difference exists, however, in how sensitivity depends on the number of reference items. It is firmly established that in static patterns the Weber fraction for numerosity discrimination decreases with reference number (Allik \& Tuulmets, 1991b; Burgess \& Barlow, 1983; Krueger, 1984; Newman, 1974). No such decrease was observed in the present set of data. On the contrary, the Observer J.A. exhibited a small but systematic increase in the Weber fraction as the number of reference items increased. Despite this discrepancy, 
the sensitivity to changes in the numerosity of dynamic events is not remarkably different from that for the static patterns. Thus, the sensitivity data provide no proof that two completely different mechanisms code the numerosity of static and of dynamic patterns.

\section{EXPERIMENT 2}

\section{Method}

Temporal events, unlike static dots, can differ by their individual duration. By definition, the number of items does not change when the duration of items becomes shorter or longer. The dependence of perceived numerosity on the spatial configuration, also irrelevant to the number of dots as such, demonstrates that logical categories are not necessarily perceptual ones. For example, it is quite probable that the perceived numerosity of dynamic events depends not simply on the number of items in the stream but on the number of simultaneously visible items. If the duration of items is increased, the expected number of dots concurrently sharing the screen also increases. To examine the effect of item duration on perceived numerosity, we used test streams with item durations shorter or longer than those in the reference stream. The duration
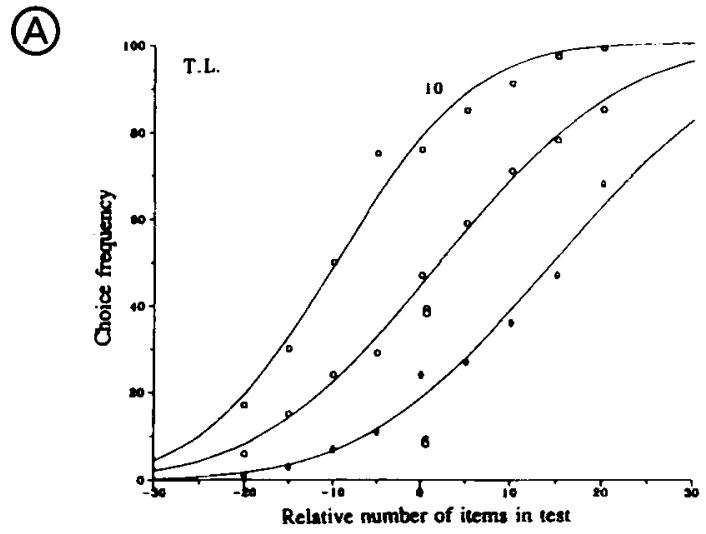

(B)

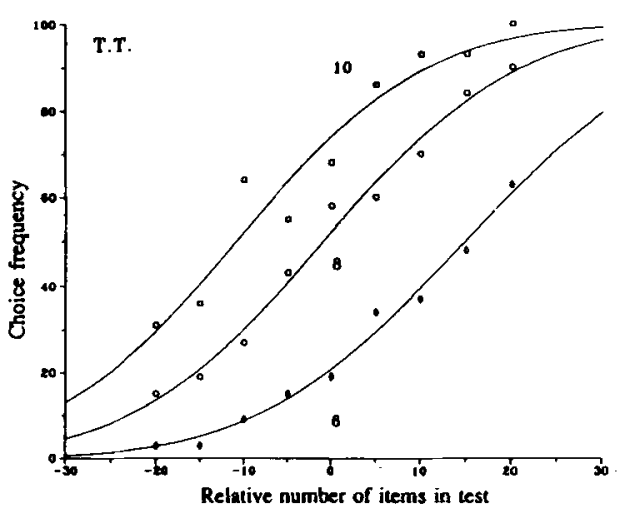

Figure 3. The choice frequency of test stimulus for 2 observers, T.L. (A) and T.T. (B), as a function of the relative number of test items $(\Delta n)$. Each curve and corresponding set of data points belongs to one of three durations of items in the test stream: $f=6,8$, and 10 frames, or 240,320 , and $400 \mathrm{msec}$, respectively. The duration of reference items was $f=8$ frames $(d=320$ msec).
Table 2

Parameter Values of the Best-Fitting Functions Shown in Figure 3

\begin{tabular}{crcrcc}
\hline Frames $(d)$ & \multicolumn{1}{c}{ CE } & \multicolumn{1}{c}{ JND } & JND $/ n$ & MSE (\%) & MAE (\%) \\
\hline \multicolumn{5}{c}{ T.L. (Figure 3A) } \\
10 & -9.7 & 8.1 & .135 & 3.68 & $4.12 *$ \\
8 & 2.0 & 10.7 & .178 & 4.17 & 2.10 \\
6 & 14.5 & 11.0 & .184 & 3.76 & 3.00 \\
& & \multicolumn{1}{c}{ T.T. (Figure 3B) } & & \\
10 & -10.9 & 11.4 & .190 & 3.93 & 1.86 \\
8 & -1.0 & 11.7 & .194 & 4.27 & 3.05 \\
6 & 14.9 & 12.3 & .205 & 3.89 & 2.23 \\
\hline
\end{tabular}

Note- $n$, number of reference items; CE, constant error; JND, just noticeable difference; JND/n, Weber fraction; MSE, mean standard error (percent); MAE, mean approximation error (percent). ${ }^{*}$ MAE > MSE. $n=60$.

of the reference items was 8 frames $(320 \mathrm{msec})$. The duration of test items was $f=6,8$, or 10 frames $(240,320$, or $400 \mathrm{msec}$, respectively); that is, one seventh, one fifth, or one fourth of the items were expected to appear concurrently. The number of reference items was $n=60$.

\section{Results}

The choice frequencies of 2 observers are plotted in Figures $3 \mathrm{~A}$ and $3 \mathrm{~B}$ as a function of the relative number of items, $\Delta n$, in the test stream. The parameters of the bestfitting function are shown in Table 2. The fit of empirical psychometric functions is satisfactory because in all but one case the MAE is smaller than the MSE. The agreement between the observers is also satisfactory.

As expected, if the duration of items was equal in both the test and the reference streams, the CE was close to zero ( 2.0 and -1.0 for T.L. and T.T., respectively). If the duration of items in the test stream exceeded that in the reference stream $(f=10)$, the whole psychometric function shifted to the left: it was necessary to remove a certain number of items from the test stream to make it look similar to the number of reference items. This shift also means that items lasting $\mathbf{4 0 0}$ msec each appear to be more numerous than items lasting only $320 \mathrm{msec}$ each. The relative shift of PSE is about -10 items for both observers, which is $16.7 \%$ of the reference level $(n=60)$. It is relevant that the duration of items in the test stream ( $400 \mathrm{msec}$ ) was $25 \%$ longer than the duration of items in the reference stream $(320 \mathrm{msec})$. If, in turn, the duration of items in the test stream was shorter $(240 \mathrm{msec})$ than that of items in the reference stream, the perceived numerosity of the test stimulus decreased, producing a rightward shift of the whole psychometric function. The relative shift of the PSE was about 15 items, which is equivalent to a $25 \%$ decrease in perceived numerosity. This decrease corresponds exactly to the percent decrease in duration.

These results indicate that it is not only difficult to distinguish the number of items from their spatial arrangement but also from the duration of items as well. The stream containing longer items appears to be more numerous. This agrees with the observation that a briefer expo- 
sure of static patterns yields an underestimation and a longer exposure yields an overestimation of the number of items in these patterns (Horne \& Turnbull, 1977; Lechelt \& Nelson, 1971). It is simplest, of course, to assume that perceived numerosity depends on the number of dots visible on the screen simultaneously. Unfortunately, this simple idea will not work. First, it does not explain why the same static pattern appears to be more numerous when it is exposed longer than when it is exposed briefly; the number of dots visible at one and the same time is equal in both cases. Second, the illusory shift in perceived numerosity does not agree quantitatively with the change in the number of dots exposed on the screen simultaneously. In the reference stream $(f=8)$, there were about 12 dots present in each frame. The decrease $(f=6)$ or increase $(f=10)$ of the item duration increased or decreased the expected number of dots per frame by 3 dots, respectively. This change is about three to five times smaller than the observed illusory shift of numerosity. The duration of items is certainly a better candidate for a stimulus attribute on which to base an impression of numerosity. But even this attribute does not explain the exact bias in the perceived numerosity.

Strictly speaking, it is impossible to change only one stimulus attribute (e.g., duration) without changing some other stimulus attributes. With the increase of duration, the total luminous energy of the item increases as well. Consequently, this experiment alone does not permit the effects of energy and duration on perceived numerosity to be distinguished. To uncouple these two effects, items in the test and reference streams in Experiment 3 had equal luminous energies but different durations.

\section{EXPERIMENT 3}

\section{Method}

To separate the duration of items from their luminous energy, we compared two streams of items with equivalent luminous energy but unequal duration of items. One of two streams was composed of light dots (L) with a luminance of $82.4 \mathrm{~cd} / \mathrm{m}^{2}$ and a duration of $80 \mathrm{msec}$. The second stream was composed of dark dots (D) having half the luminance of the bright ones $\left(41.2 \mathrm{~cd} / \mathrm{m}^{2}\right)$ but twice the duration $(160 \mathrm{msec})$. The total luminous energy in these two items was equal. In the critical stimulus condition, the reference stream was composed of light dots and the test stream of dark ones (LD). In addition, two other conditions were added in which both streams were composed of either light (LL) or dark (DD) dots; that is, the luminance and duration of items in the test and reference streams were equal. A reduced number (seven) of test item values, $\Delta n$, was used in these two conditions. The number of items in the reference stream was $n=100$.

\section{Results}

The results are shown in Figures 4A (T.L.) and 4B (T.T.), and numerical values of the fit are presented in Table 3. As expected, there was no difference between the two cases (LL, DD) in which luminance and duration were equal in both streams. The psychometric functions were fitted simultaneously, using both sets of data, as indicated by squares (LL, light dots) and triangles (DD, dark dots).
With long dark test dots and short bright reference dots (LD), the psychometric function is considerably shifted to the left, the $\mathrm{CE}$ being equal to -17.6 (T.L.) and -24.6 (T.T.). Thus, with energy held constant, the effect of duration still endured-the stream containing longer items appears to be more numerous. However, the change in perceived numerosity was proportionally smaller than the change in duration. In principle, this discrepancy can be explained by the difference between perceived and physical durations. Efron (1970) demonstrated that the time interval between the adjusted onset and offset times of a flash longer than $130 \mathrm{msec}$ corresponds to its actual duration. For briefer stimuli, the perceived duration outlasts the actual duration. On the other hand, perceived duration depends not only on duration but on intensity as well. The relationship between intensity and perceived duration is a controversial issue; in some studies a direct relationship between these two variables has been found, but

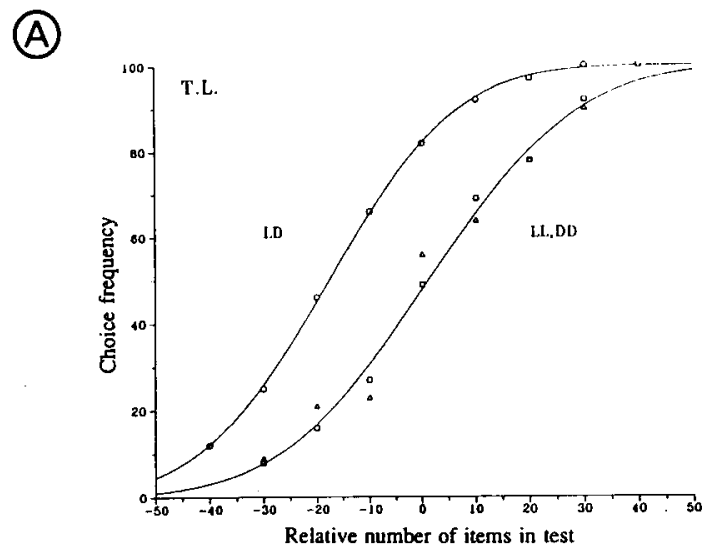

(B)

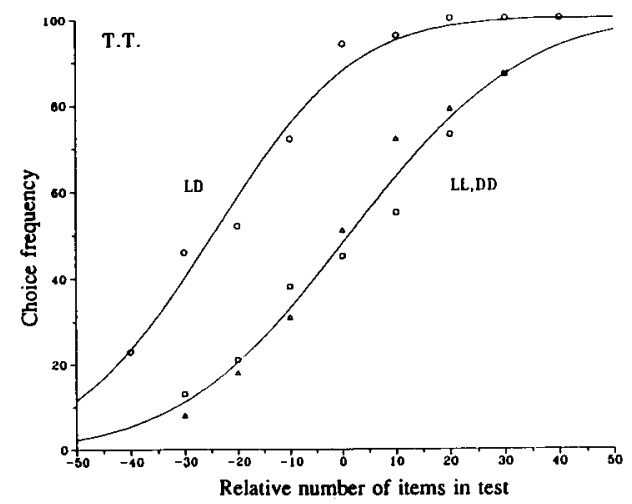

Figure 4. The choice frequency for different luminance and duration of items for Observers T.T. (A) and T.T. (B). The test and reference streams contained items equated in luminous energy. Streams were composed of either light dots (L) with $82.4 \mathrm{~cd} / \mathrm{m}^{2} \mathrm{lu}$ minance $(d=80 \mathrm{msec})$ or dark dots (D) with half luminance $\left(41.2 \mathrm{~cd} / \mathrm{m}^{2}\right)$ but twice the duration $(d=160 \mathrm{msec}) . \mathrm{LL}$, test and reference streams composed of light dots (circles); DD, dark dots (squares); LD, reference composed of light dots and stimulus of dark dots (triangles). LL and DD data points were approximated by a single curve. 
Table 3

Parameter Values of the Best-Fitting Functions Shown in Figure 4

\begin{tabular}{|c|c|c|c|c|c|}
\hline Type & $\mathrm{CE}$ & JND & $\mathrm{JND} / \boldsymbol{n}$ & MSE (\%) & $\operatorname{MAE}(\%)$ \\
\hline \multicolumn{6}{|c|}{ T.L. (Figure 4A) } \\
\hline $\begin{array}{l}\text { DD,LL } \\
\text { DL }\end{array}$ & $\begin{array}{r}1.1 \\
-17.7\end{array}$ & $\begin{array}{l}14.8 \\
12.9\end{array}$ & $\begin{array}{l}.148 \\
.128\end{array}$ & $\begin{array}{l}4.03 \\
3.36\end{array}$ & $\begin{array}{l}3.63 \\
0.57\end{array}$ \\
\hline \multicolumn{6}{|c|}{ T.T. (Figure 4B) } \\
\hline $\begin{array}{l}\mathrm{DD}, \mathrm{LL} \\
\mathrm{DL}\end{array}$ & $\begin{array}{r}1.3 \\
-24.6\end{array}$ & $\begin{array}{l}17.3 \\
14.3\end{array}$ & $\begin{array}{l}.173 \\
.143\end{array}$ & $\begin{array}{l}4.21 \\
3.29\end{array}$ & $\begin{array}{l}4.05 \\
3.89 *\end{array}$ \\
\hline
\end{tabular}

Note- $n$, number of reference items; CE, constant error; JND, just noticeable difference; JND/n, Weber fraction; MSE, mean standard error (percent); MAE, mean approximation error (percent). D, dark dots; $L$, light dots. $n=100$. *MAE $>$ MSE.

others have resulted in an inverse relationship (Nisly \& Wasserman, 1989). Nisly and Wasserman found that when the subject had to indicate the moment when all traces of the sensation had disappeared, a direct relationship was obtained. If the same logic is valid for the numerosity discrimination task, the short but more intensive items ought to be perceived to outlast their physical duration. Thus, we may exclude that perceived numerosity corresponds to the perceived duration of items, which, in turn, depends on their duration and intensity.

\section{EXPERIMENT 4}

\section{Method}

As with the static case, the distribution of items in a spatiotemporal volume can be made either inhibitory or satellite. Again, the expected separation between two neighboring items can be larger or smaller than in the case of the unrestricted bombardment. In Experiment 4 , we tested whether inhibitory overestimation and the satellite underestimation exist in the spatiotemporal domain as well.

All items in this experiment lasted $d=80 \mathrm{msec}$; on the average, $5 \%$ of the items were shown at one time. The reference stream consisted of $n=100$ reference items, distributed randomly across the available set of spatiotemporal locations. Three different random processes were used to generate the test stimuli.

Random process. The test stimuli were identical to the reference stimuli, except that the number of items varied systematically (seven different values) around the reference value.

Inhibitory process. The second type of stream was generated so that no two adjacent items could be closer to each other than 3 positions $\left(0.278^{\circ}\right)$ vertically or horizontally and 3 frames (240 msec) temporally. The spatial inhibitory distance was chosen on the basis of the previous study, which estimated the radius, $R$, in which every dot has an influence on its surrounding, to be about $0.3^{\circ}$ (Allik \& Tuulmets, 1991a).

Satellite process. Half the items in the display (parents) were randomly distributed across available spatiotemporal locations, but the other half were each assigned to a parent item and were positioned randomly within an imaginary $7 \times 7 \times 7$ spatiotemporal cubicle $\left(0.651^{\circ} \times 0.651^{\circ}\right.$ spatial window, seven frames long) centered about the parent item. All positions, except those already occupied and positions next to them, could be chosen equiprobably. The distance between satellites (i.e., parent and assigned items) in the horizontal or vertical direction was no larger than $0.31^{\circ}$, and the temporal separation was from 80 to $240 \mathrm{msec}$. The average distance between two satellite items was about $0.25^{\circ}$ and $160 \mathrm{msec}$.

\section{Results}

The test choice frequencies of 2 observers for three different random processes are plotted in Figures $5 \mathrm{~A}$ and
5B as a function of the relative number of test items, $\Delta n$. The best fit is graphically shown by continuous curves, and the corresponding numerical values are presented in Table 4.

For the random process, the PSE was close to the actual number of reference items. The other two psychometric functions, however, were shifted in two opposite directions. The $C E$ values corresponding to the inhibitory process were negative, -11.5 (T.L.) and -8.0 (T.T.). Thus, about 90 items distributed according to the inhibitory process appeared to be as numerous as 100 randomly distributed items. The apparent shift in numerosity for the satellite process also consisted of about 10 items but in the positive direction ( $\mathrm{CE}=9.7$ and 12.0, respectively). Thus, the number of spatiotemporal items distributed according to the satellite process was underestimated, and some number of additional items was required to make them appear as numerous as the reference ones. It is also notable that the JND is smaller for the inhibitory process than for the random process and that it is larger for the satellite process. Burgess and Barlow (1983) demonstrated that two inhibitory distributions of dots are discriminated more precisely than two completely random arrays of dots. The better discrimination of inhibitory distributions and poorer discrimination of satellite distributions can be explained in terms of the occupancy model: the same increase in the number of dots leads to a more salient change
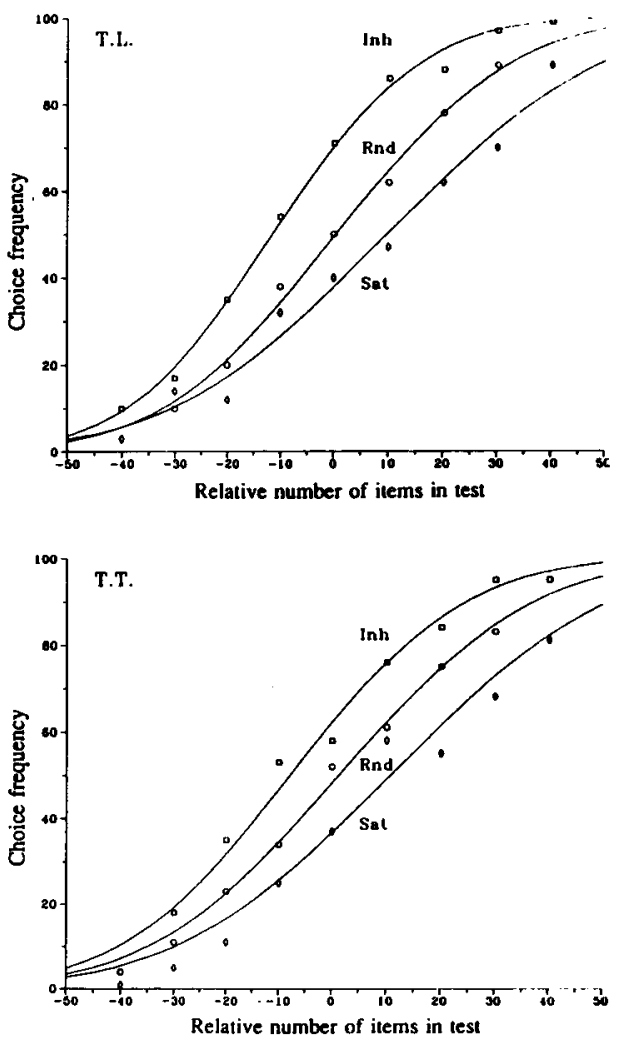

Figure 5. The choice frequency for various spatiotemporal distributions of items as a function of $\Delta \mathbf{n}$. The test stimulus was generated according to the random (Rnd), inhibitory (Inh), or satellite (Sat) process (circles, squares, and rhombus, respectively). 
Table 4

Parameter Values of the Best-Fitting Functions Shown in Figure 5

\begin{tabular}{|c|c|c|c|c|c|}
\hline Type & $\mathrm{CE}$ & JND & $\mathrm{JND} / n$ & MSE (\%) & MAE (\%) \\
\hline \multicolumn{6}{|c|}{ T.L. (Figure 5A) } \\
\hline $\begin{array}{l}\text { Random } \\
\text { Inhibitory } \\
\text { Satellite }\end{array}$ & $\begin{array}{r}0.3 \\
-11.5 \\
9.7\end{array}$ & $\begin{array}{l}17.2 \\
14.5 \\
21.2\end{array}$ & $\begin{array}{l}.172 \\
.145 \\
.212\end{array}$ & $\begin{array}{l}4.21 \\
3.62 \\
4.09\end{array}$ & $\begin{array}{l}2.00 \\
2.00 \\
4.00\end{array}$ \\
\hline \multicolumn{6}{|c|}{ T.T. (Figure 5B) } \\
\hline $\begin{array}{l}\text { Random } \\
\text { Inhibitory } \\
\text { Satellite }\end{array}$ & $\begin{array}{r}1.2 \\
-8.0 \\
12.0\end{array}$ & $\begin{array}{l}19.0 \\
17.2 \\
20.8\end{array}$ & $\begin{array}{l}.190 \\
.172 \\
.208\end{array}$ & $\begin{array}{l}4.34 \\
3.83 \\
4.02\end{array}$ & $\begin{array}{l}1.87 \\
3.70 \\
4.76^{*}\end{array}$ \\
\hline
\end{tabular}

Note- $n$, number of reference items; CE, constant error; JND, just noticeable difference; JND $/ n$, Weber fraction; MSE, mean standard error (percent); MAE, mean approximation error (percent). $n=100$. *MAE > MSE

of the occupancy value in an inhibitory distribution and to a less prominent one in a satellite distribution (cf. Allik \& Tuulmets, 1991a).

As the results of this experiment demonstrate, inhibitory overestimation and satellite underestimation occur not only for static dot patterns but also for series of items distributed over a spatiotemporal interval. Thus, two spatially and temporally proximate items have less total impact on the numerosity decision than do two distant ones. Two items in the inhibitory distribution cannot be closer than a certain spatial and temporal interval. In the random distribution, however, there is a certain probability that two items will be in close proximity with each other. In the satellite distribution, in turn, each item had at least one neighbor not very far from it. The attenuation of the impact with proximity is probably simply another way of saying that two items mask each other if they are close enough in space and time. In our previous study, we found that perceived numerosity decreases with spatial proximity of dots (Allik \& Tuulmets, 1991a). At the same time, it is rather well documented that the number of successive objects that appear to be physically present at the same time decreases with distance between adjacent objects (Allport, 1968; Di Lollo \& Dixon, 1988; Di Lollo \& Hogben, 1987; Dixon \& Hammond, 1972; Farrell, 1984).

If perceived numerosity is related to the apparent duration of items, as proposed above, the observed satellite underestimation can be explained at least in principle in terms of the attenuation of apparent duration produced by the spatial proximity of items. Unfortunately, the present data do not permit us to separate the contributions of spatial and temporal proximities on perceived numerosity. Conceivably, only the spatial proximity of items is important and the temporal proximity is rather irrelevant for the formation of an impression about numerosity. Is it possible, in principle, to distinguish the spatial impact from the temporal one? Space and time do not embrace the concept of physical nearness independently. Although two items may appear exactly in one and the same place, they are not close to each other if there is a considerable time interval between their occurrences. Two items can be proximate only if they are close in space and time simultaneously. Therefore it only makes sense to speak about the relative weight of spatial and temporal proximity on the impression of numerosity.

\section{EXPERIMENT 5}

\section{Method}

The main idea behind Experiment 5 was as follows. The relative contribution of spatial and temporal nearness of items can be estimated if, when one factor, spatial or temporal distance, is varied and the second factor is held constant. If one of these two variables is irrelevant for satellite underestimation, the numerosity bias will disappear or decrease considerably in that particular condition. On the other hand, if each of these two conditions can in isolation produce satellite underestimation, approximately the same amount of numerosity underestimation will be produced when these two variables are varied jointly. According to the simplest rule, the underestimation in the joint condition can be predicted from a composition of numerosity biases obtained in conditions in which space and time separation were varied separately.

The duration and the number of reference items were the same as in the previous experiment, $d=80$ and $n=100$. There were four types of test stimuli. The first was generated by a completely random process, and it serves as a basis of comparison for the other three processes, which all were of the satellite type. Half of all items were positioned randomly, and the remaining satellite dots were assigned to their respective parents.

In the spatiotemporal condition, any location within a $7 \times 7$ spatial window and 7 -frame-long temporal segment, both centered at the parent item, could be chosen except the location of the parent item itself and the location next to it. This was one of the test stimulus types (satellite process) used in the previous experiment.

In the spatial condition, the two satellite items (i.e., parent and assigned items) always started and ended at the same time; only spatial location within a $7 \times 7$ window was varied. The average distance between the parent item and its satellite was 2.7 positions or about $0.25^{\circ}$.

Finally, in the temporal condition, the two satellites always occupied the same spatial location on the display. The interval between the parent item and its temporal satellite was from 1 to 3 frames $(80-240 \mathrm{msec})$. The average temporal separation between the parent and satellite was $160 \mathrm{msec}$.

\section{Results}

The choice frequencies of 2 observers for four stimulus types (random and three satellite processes) is plotted in Figure 6 as a function of the relative number of test items, $\Delta n$. The corresponding fitted values are shown in Table 5.

As in the previous experiment, the psychometric function of the random process (filled circles) was centered about zero and all curves representing satellite processes were shifted 5-10 units to the right. All types of satellite processes-the spatiotemporal (stars, dotted curve), spatial (squares, short dashes), and temporal (triangles, long dashes)-reduced the perceived number of items approximately equally. This reduction was, as in the previous experiment, accompanied by a decrease in the precision of discrimination, with the largest decrease in the spatial condition.

The satellite underestimation was observed in all three cases, including two in which the satellite distance varied only in space or in time. Consequently, both components determining the physical nearness of two items affected the perceived numerosity of those items. The spatiotem- 
poral satellite process evidently was a compound of two other processes in which spatial and temporal separation between satellites varied in isolation. As expected, the perceived numerosity of the compound process can be predicted from the perceived numerosity of the isolates. For example, the CE of Observer J.A. was 9.6 and 6.2 in the spatial and temporal conditions, respectively. According to the simplest multiplicative or additive rules of averaging, the expected change in the composite spatiotemporal condition would be about 7.7 or 7.9 -which is rather close to the observed 7.9. For the second observer, T.T., however, the predicted shift of 5.8 was a little bit smaller than the observed 8.3 shift in the perceived number. This discrepancy may be due to many factors, including error in the determination of the exact value of PSE. Also, the appearance of patterns may have caused some response biases. In spite of this discrepancy, it is quite obvious that perceived numerosity decreases with both spatial and temporal proximity between the visual items.
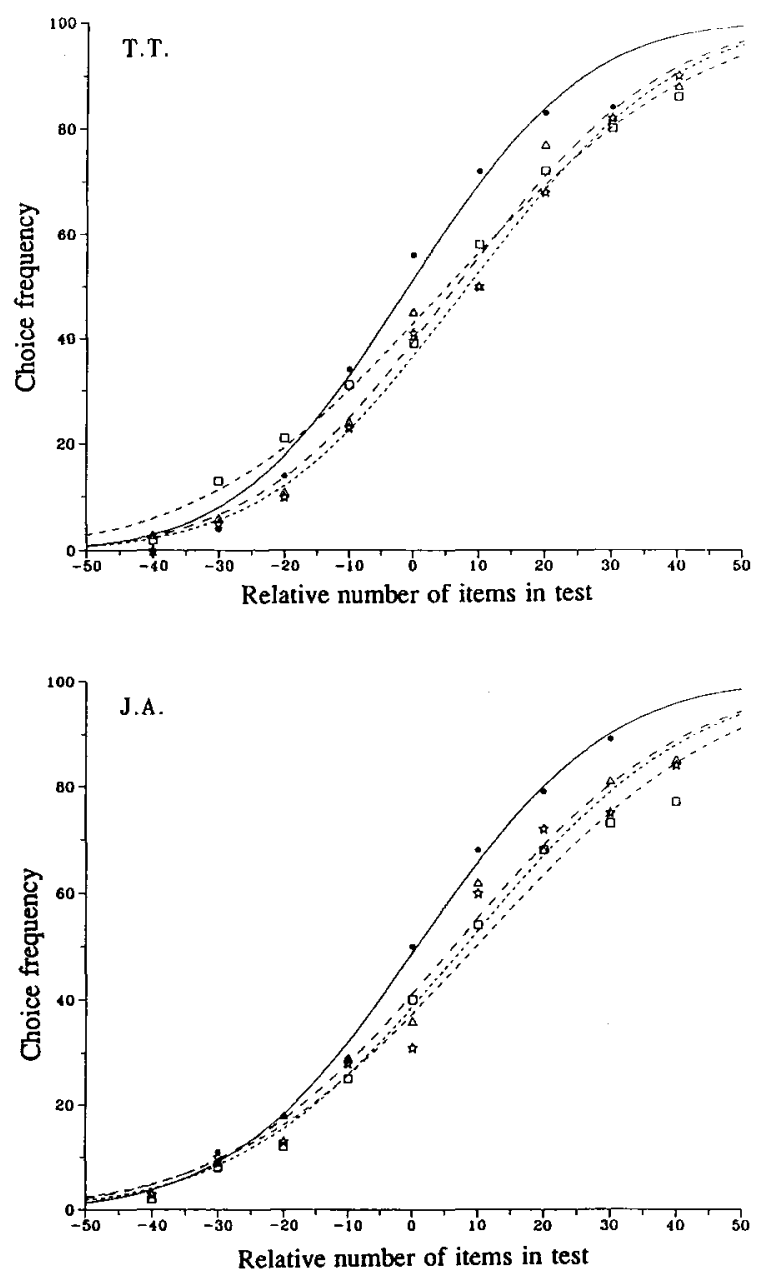

Figure 6. The choice frequency for the random process (filled circles) and three types of satellite process: spatiotemporal (stars), spatial (squares), and temporal (triangles). The best fit to the random process data is shown by the continuous curve and to the three others by broken lines (the length of dashes increases in the order of the satellite processes given above).
Table 5

Parameter Values of the Best-Fitting Functions Shown in Figure 6

\begin{tabular}{|c|c|c|c|c|c|}
\hline Type & $\mathrm{CE}$ & JND & $\mathrm{JND} / n$ & $\operatorname{MSE}(\%)$ & $\operatorname{MAE}(\%)$ \\
\hline \multicolumn{6}{|c|}{ T.T. (Figure 6A) } \\
\hline Random & -0.7 & 14.1 & .141 & 3.98 & $4.45^{*}$ \\
\hline Spatiotemporal & 8.3 & 16.3 & .163 & 3.74 & 2.07 \\
\hline Spatial & 5.2 & 19.7 & .197 & 4.05 & 2.41 \\
\hline Temporal & 6.5 & 16.4 & .164 & 3.79 & 3.68 \\
\hline \multicolumn{6}{|c|}{ J.A. (Figure 6B) } \\
\hline Random & 0.7 & 15.5 & .155 & 4.11 & 1.76 \\
\hline Spatiotemporal & 7.9 & 18.6 & .186 & 3.96 & $4.44^{*}$ \\
\hline Spatial & 9.6 & 20.3 & .203 & 4.03 & 3.75 \\
\hline Temporal & 6.2 & 18.8 & .188 & 3.98 & 3.18 \\
\hline
\end{tabular}

Note- $n$, number of reference items; CE, constant error; JND, just noticeable difference; JND/n, Weber fraction; MSE, mean standard error (percent); MAE, mean approximation error (percent). $n=100$. *MAE > MSE.

\section{DISCUSSION}

The events whose numerosity was estimated were dots appearing at various times in various positions on the screen and then disappearing some time later. This kind of dynamic stimulation contrasts with the use of static patterns in previous studies. The results of this study demonstrated, however, that the perception of the numerosity of dynamic items did not differ drastically from that of static patterns. First, numerosity discrimination was comparably precise in both conditions. Second, two fundamental numerosity biases, inhibitory overestimation and satellite underestimation, that were known to occur for dot patterns, were also found for streams of dynamic events. It was also demonstrated that both spatial and temporal proximities between items in the stream of dynamic events are responsible for the numerosity bias.

Formally, at least, the number of items does not change when the distance between items changes or when the duration of items becomes shorter or longer. Nor is there room to doubt that the observer can readily understand the instruction to estimate the number of items. If there were enough time for the direct counting of items, the answers would certainly be close to veridical. In a real experimental situation, however, relying only on the immediate impression of numerosity, the estimates were neither very precise nor unbiased. Many stimulus attributes, formally irrelevant to numerosity as such, affect the judgment of numerosity. Typically, the influence of irrelevant attributes is regarded as erroneous, like the errors of a physical instrument designed to measure, for example, current, but which exhibits undesired sensitivity to temperature and humidity. Alternatively, as was argued in the introduction, the biases may reflect the inability to measure relevant stimulus attributes that are required to complete a given visual task. Instead of the proper stimulus attribute, another attribute, one that is only indirectly related to the actual number of items, may have been estimated.

The only sufficiently elaborated model that can explain various numerosity biases occurring in static patterns, not 
merely in qualitative but in strictly quantitative terms, is the occupancy model (Allik \& Tuulmets, 1991a). Occupancy (as was briefly described in the introduction) is the basis on which the observer makes his or her decisions about numerosity. This attribute does not require a sophisticated device to measure it. The only thing that is needed is a set of equal-sized disks. Each dot in the pattern must be covered by one such disk, after which the plane area covered by all the disks has to be estimated in some way. If the number of dots is kept constant, the area covered by the disks can be changed only by altering the amount of overlap between disks. It was surprising (and gratifying!) that this elementary measure accurately predicted not only the sign of all known numerosity biases, but their exact choice probabilities. Is it possible, at least in principle, to extend the occupancy model to a more general spatiotemporal case? In the present formulation, the influence each dot exerts on its neighborhood spreads in the constant circular radius $R$ of the picture plane. Inhibitory overestimation and satellite underestimation in dynamic patterns indicate that the influence spreads not only in space but also in time. Thus, each dot has an influence on a certain spatiotemporal surrounding. More formally, the impact of an element is presumed to be distributed in the constant radius $R$, which is a function of one temporal $(t)$ and two spatial $(x, y)$ arguments: $R=\left(\alpha x^{2}+\beta y^{2}+\gamma t^{2}\right)^{1 / 2}$, where $\alpha, \beta$, and $\gamma$ are the coefficients of propagation in the respective dimension.

This generalization seems intuitively plausible. Each item is posited to occupy a spherical volume in threedimensional space-time. The total impression of numerosity of items is determined by the amount of space-time apparently occupied by these items. If in space it depends on a plane area apparently occupied by dots in the temporal domain, it obviously depends on the apparent duration of an event. Experiments 2 and 3 also support this interpretation; the perceived numerosity depends on the duration of items, not their contrast or total luminance energy. It was quite sensible to propose that the luminous energy of items may affect the perceived numerosity of those items. There is evidence that energetically stronger items have a larger impact on perceived numerosity than do the same number of energetically weaker items (cf. Lechelt, 1974b). The role of luminous energy in the salience of the perceived orientation, motion, and depth is also well documented (Allik, in press; Foley, 1976; Nishida \& Takeuchi, 1990; Prazdny, 1984). But even when two groups of items were equated in luminous energy, the group having more durable components appeared more numerous. It is interesting that the converse may be also true: the perceived duration of an item depends on the number of dots in it (Mo, 1974, 1975).

The amount of space-time occupied by an item, however, can be modified by changing the spatio-temporal nearness of items. If two items are close enough, their individual territories overlap, and the total impact on the impression of numerosity decreases in proportion to this overlap. In spite of its plausibility, the updated occupancy model was not tested quantitatively. The limited number of test conditions used in this study made it very improbable that the updated occupancy model could not be fitted satisfactorily to the data.

Given that the proposed simple generalization of the occupancy model is valid, a question about its relation to other possible interpretations arises. Does the occupancy model exclude other interpretations, particularly in terms of certain psychological mechanisms? In particular, as reviewers of an earlier version of this paper proposed, some results presented in this study can be interpreted in terms of "iconic memory." The explanation in terms of the space-time occupancy index is not incompatible with many other more specific psychological theories, including the inexactly formulated concept of iconic memory. It is quite probable, for example, that the spread of influence in the temporal domain is nothing but another word for visual masking. The impact of two items decreases if they are temporally close to each other. The occupancy model, like its possible augmentations, is a strictly psychophysical construction, specifying a stimulus attribute that serves as a basis for the decisions about numerosity. There are many ways in which this stimulus attribute can be extracted and processed. Although a processing explanation is desirable in many cases, it is often only a less precise reformulation of some exact psychophysical relation.

One of the basic findings of this study was that both spatial and temporal nearness affect perceived numerosity. This means, in particular, that space and time are interchangeable and that the amount of change in perceived numerosity caused by spatial proximity of items can be produced by appropriately chosen temporal intervals between items. This conclusion about interchangeability of spatial and temporal coordinates is supported by our previous result: in the discrimination of numerosity of two streams of visual events, it is irrelevant whether the change in the total number of test items is achieved by adding simultaneous items to one frame or by increasing the number of succeeding frames (Allik \& Tuulmets, 1991b). A similar kind of space-time interchangeability is known for other perceptual phenomena: perceived motion (Korte's laws), estimation of space and time intervals (kappa and tau effects), and so forth.

There seems to be a basic difference between the extraction of geometric and nongeometric information from an image. For example, the extraction of nongeometric information typically does not vary with exposure duration. In contrast, the identification of geometric properties improves progressively with exposition time (Watt, 1987). In this respect, it is interesting that the detectability of stationary or moving target dot forms embedded in a background of a spatiotemporally varying noise dot pattern was found to decrease linearly with both spatial and temporal separation between the target dots (Falzett \& Lappin, 1983). There is no doubt that both form and motion require refined geometrical notions for their specification. The judgments about numerosity, on the contrary, are based on very primitive geometric information. Nevertheless, perceived numerosity, as it is explicated in 
the occupancy index, is unquestionably a nonlinear function of spatial and temporal separation between target dots. But despite this nonlinearity, spatial and temporal proximity may still have an interchangeable effect on perceived numerosity.

\section{REFERENCES}

ALLIK, $\mathcal{J}$. (in press). Resolving ambiguities in orientation, motion and depth domains. Perception.

Allik, J., \& Tuulmets, T. (1991a). Occupancy model of perceived numerosity. Perception \& Psychophysics, 49, 303-314.

Allik, J., \& TUUlmets, T. (1991b). The perception of visual numerosity. In R. Watt (Ed.), Vision and visual dysfunctions: Patter recognition by man and machine (Vol. 14, pp. 125-142). London: Macmillan.

AluIK, J., Tuulmets, T., \& Vos, P. G. (1991). Size invariance in visual number discrimination. Psychological Research, 53, 290-295.

AllporT, D. A. (1968). Phenomenal simultaneity and the perceptual moment hypothesis. British Joumal of Psychology, 59, 395-406.

BURGESS, A., \& BARLOW, H. B. (1983). The precision of numerosity discrimination in arrays of random dots. Vision Research, 23, 811-820.

Diggle, P. J. (1983). Statistical analysis of spatial patterns. London: Academic Press.

Di Lollo, V., \& Dixon, P. (1988). Two forms of persistence in visual information processing. Journal of Experimental Psychology: Human Perception \& Performance, 14, 671-681.

Di Lollo, V., \& HogBen, J. H. (1987). Suppression of visible persistence as a function of spatial separation between inducing stimuli. Perception \& Psychophysics, 41, 345-354.

Dixon, N. F., \& Hammond, E. J. (1972). The attenuation of visual persistence. British Journal of Psychology, 63, 243-254.

EFRON, R. (1970). The relationship between the duration of a stimulus and the duration of perception. Neuropsychologia, 8, 37-55.

FALZETT, M., \& LAPPIN, J. S. (1983). Detection of visual forms in space and time. Vision Research, 23, 181-189.

FARRELL, J. E. (1984). Visible persistence of moving objects. Journal of Experimental Psychology: Human Perception \& Performance, 10, 502-511.

FoLEY, J. M. (1976). Binocular depth mixture. Vision Research, 16, 1263-1267.

GiNSBURG, N. (1976). Effect of item arrangement on perceived numerosity: Randomness vs regularity. Perceptual \& Motor Skills, 43, 663-668

Ginsburg, N., \& Goldstein, S. R. (1987). Measurement of visual cluster. American Journal of Psychology, 100, 193-203.

Horne, E. P., \& TuRnbull, C. E. (1977). Variables of color, duration, frequency, presentation order, and sex in the estimation of dot frequency. Journal of General Psychology, 96, 135-142.

HUNTER, W. S., \& Sigler, M. (1940). The span of visual discrimination as a function of time and intensity of stimulus. Joumal of Experimental Psychology, 26, 160-179.

Krueger, L. E. (1984). Perceived numerosity: A comparison of magnitude production, magnitude estimation, and discrimination judgments Perception \& Psychophysics, 35, 536-542.

LeCHELT, E. C. (1974a). Pulse number discrimination in tactile spatiotemporal patterns. Perceptual \& Motor Skills, 39, 815-822.

LECHELT, E. C. (1974b). Stimulus intensity and spatiality in tactile temporal numerosity discrimination. Perception. 3, 297-302.

LECHELT, E. C., \& NELSON, T. M. (1971). Numerosity discrimination under varying conditions of steady illumination: $A$ temporal analysis of a spatial factor. Journal of General Psychology, 84, 121-132.

Luccıo, R. (1983). La percepzione della numerosità. Storia e Critica della Psicologia, 4, 215-283.

Mo, S. S. (1974). Comparative judgment of temporal duration as a function of numerosity. Bulletin of the Psychonomic Society, 3, 377-379.

Mo, S. S. (1975). Temporal reproduction of duration as a function of numerosity. Bulletin of the Psychonomic Society, 5, 165-167.

Morgan, M. J., Hole, G. J., \& Glennerster, A. (1990). Biases and sensitivities in geometrical illusions. Vision Research, 30, 1793-1810.

Newman, C. V. (1974). Detection of differences between visual texures with varying number of dots. Bulletin of the Psychonomic Society. 4, 201-202.

Nishida, S., \& TAKEUCHI, T. (1990). The effect of luminance of affinity of apparent motion. Vision Research, 30, 709-721.

NisLY, S. J., \& WASSERMAN, G. S. (1989). Intensity dependence of perceived duration: Data, theories, and neural integration. Psychological Bulletin, 106, 483-496.

Prazdny, K. (1984). On the perception of Glass patterns. Perception. $13,469-578$.

RIPLEY, B. D. (1981). Spatial statistics. New York: Wiley.

VIVIANI, P. (1979). A diffusion model for discrimination of temporal numerosity. Journal of Mathematical Psychology, 19, 108-136.

WATT, R. J. (1987). Scanning from coarse to fine spatial scales in the human visual system after the onset of a stimulus. Joumal of the Optical Society of America A, 4, 2006-2021.

\section{NOTE}

1. The method of the direct approximation of psychometric functions was proposed by Sverker Runeson. It uses the downhill simplex method to minimize misprediction of two independent variables of the psychometric function, the mean and the standard deviation. In the present study, the squared deviation between observed and predicted choice probability was minimized. The minimization program was written in $C$ and is a slightly modified version of the QuickBASIC program that was kindly provided by Runeson.

(Manuscript received December 4, 1991; revision accepted for publication September 23, 1992.) 